\title{
Gender and ploidy in cancer survival
}

\author{
Susanne Schulze $\cdot$ Iver Petersen
}

Accepted: 13 September 2010 /Published online: 22 March 2011

(C) The Author(s) 2011. This article is published with open access at Springerlink.com

\begin{abstract}
Background Females carry a better prognosis than men for many cancer types. We hypothesized that chromosomal changes, in particular numerical alterations of the sex chromosomes or the presence of near-triploidy may contribute to these gender differences.

Methods To characterize the influence of gender a literature search was performed for survival data of 27 tumor types. All entities were categorized by the strength of evidence for differences in survival between females and males. To test our hypothesis the Mitelman database of chromosomal alterations was evaluated for the major tumor types occurring in both women and men. Numerical gonosome alterations were documented and mean chromosome numbers were converted into histograms to provide insight into the ploidy level of 37 cancer types.

Results In general, a survival advantage of women could be shown for most, but not all cancer types. In addition, 36.859 karyograms were analyzed. Numerical gonosome alterations were more frequent in males than females indicating a potential link with gender differences in survival. Neartriploidy was a common phenomenon in many cancer types suggesting that it represents a metastable condition of the cancer genome. It was not related to gender differences in survival. However, the extent of triploidy and aneuploidy was associated with poor prognosis in carcino-
\end{abstract}

Electronic supplementary material The online version of this article (doi:10.1007/s13402-011-0013-0) contains supplementary material, which is available to authorized users.

S. Schulze $\cdot$ I. Petersen $(\square)$

Institute of Pathology, Universitätsklinikum Jena,

Ziegelmühlenweg 1,

07740 Jena, Germany

e-mail: iver.petersen@med.uni-jena.de mas. There was no single case in the Mitelman database with normal chromosome number $(n=46)$ that did not carry at least one structural or numerical aberration.

Conclusions Our study highlights the importance of chromosomal changes in tumor formation and progression. In addition, it suggests potential associations with gender specific differences in survival.

Keywords Gender · Aneuploidy · Chromosomal chaos · Prognosis

\section{Introduction}

Observations showed that chromosomal alterations and aneuploidy are associated with the survival of tumor patients. Furthermore, it is known that there is an influence of sex on tumor survival. In this work, on the one hand, we wanted to systematically investigate whether and if so in which tumor types gender has an influence on the prognosis. As a predictive value we used the 5-year survival rate to compare the difference in survival between men and women. On the other hand the influence of ploidy on tumor survival should be investigated.

The term ploidy is related to the chromosome complement, but also to the DNA content of cells. Aneuploidy is the state of a cell in which there are numerical aberrations of chromosomes compared to normal. Aneuploidy may also occur when structural chromosomal aberrations are associated with the gain or loss of genetic material. These genetic imbalances are often detected by a change in the DNA content of cells [29]. Overall, aneuploidy is a common and essential characteristic of malignant tumors [20]. Some authors relate the state of aneuploidy exclusively to 
numerical chromosomal aberrations while others include structural aberrations that are associated with the loss or gain of chromosomal material [16].

In this paper we used the term aneuploidy primarily in the context of numerical chromosomal aberrations. A correlation between aneuploidy and in particular the extent of chromosomal changes with the survival of cancer patients has already been shown long time ago [22]. However, in most studies only one tumor was examined for aneuploidy. There are only few studies which analyzed several tumor entities [5], [72].

Malignant tumors frequently exhibit DNA distributions in the near triploid range as we recently pointed out for lung cancer [60]. Triploidy in cancer can be regarded as evidence for aneuploidy. It is yet unclear whether this state of triploidy is related to specific biological or clinicpathological tumor characteristics.

Specifically, we asked ourselves in this work the following questions: 1) Which tumor entities show gender differences in survival? 2) Which tumor types harbour aneuploid chromosome numbers and have a near triploid chromosome set? 3) Is there a correlation between triploidy/ aneuploidy and survival? 4) Is there a correlation between the ploidy level/triploidy or sex chromosome aberrations and gender-specific difference in cancer survival? More than 30 tumor entities were analyzed regarding their survival data and ploidy state.

\section{Materials and methods}

\subsection{Gender-specific prognosis}

In this work we examined whether gender could have an influence on the prognosis. As a predictive value, we used the 5-year survival rate to compare the difference in survival between men and women. We examined the overall survival of 34 tumor entities, including seven gender-specific tumor types. Data on gender-specific prognosis were retrieved from PubMed. We took into account preferentially studies in which the entire tumor entity was represented, i.e. no studies in which only certain stages or tumor subgroups (age, etc.) were considered.

In this respect, there are only few sources that reported comprehensively on multiple tumor entities. Our main sources of the survival rate were the Robert Koch Institute (RKI) and the Eurocare-3 study, this data mainly covered European and German populations.

In addition, many studies on individual tumor entities were retrieved in PubMed using the following search parameters: tumor entity, such as "lung cancer", "epidemiology", "sex specific survival" or "gender-specific prognosis". The studies were then summarized in a table in which the evidence for gender-specific difference in prognosis is reflected by the number of studies reporting such differences (Table 1).

\subsection{Tumor ploidy}

To search for the ploidy in various tumors, we used the Mitelman database [44]. It represents only modal cancer cell lines and not all existing sidelines that are present in these tumors. With the option "Full Searcher" specific tumor types can be selected. The result is a table that lists the complete karyotypes of the different tumor cases. These karyotypes were transferred to Excel. For each karyotype, we recorded the total chromosome numbers. In some tumors, only a range of chromosome numbers were available (e.g. 64-70). For these cases a mean value was calculated, i.e. minimum plus maximum chromosome number divided by two, to get a single value for each case (i.e. 67 in the above mentioned example). The range of the chromosome numbers of these cases are available as supplementary data. Importantly, only three cases had numbers that varied between 46 (minimum) and 92 (maximum) chromosomes (1 colon carcinoma, 1 multiple myeloma, 1 thyroid carcinoma). These cases were eliminated from the ensuing analysis.

The numbers were then presented in the form of a histogram (Fig. 1). To simplify the representation, the tumors were grouped according to a range including cases with three adjacent chromosome numbers. That means for example that within the group of diploid tumors not only those cases with 46 chromosomes but also those with 45 and 47 were included. In general, the histograms represent the distribution of chromosome numbers within a tumor entity.

In order to define distinct ploidy states, the proportions of cases with hyperdiploid, predominant triploid, predominant hypertriploid and aneuploid sets of chromosomes were calculated. "Hyperdiploid" tumors were classified as those with chromosome numbers larger than $47(n>47)$. Accordingly, cases with "predominant triploid" karyotpyes were defined as tumors with chromosome numbers from 57 to $83(57<\mathrm{n}<83)$. "Predominantly hypertriploid" tumors were those with $n>57$. "Aneuploid" tumors were classified as those with chromosome numbers not in the diploid or tetraploid range ( $n \neq 45-47$ and $90-92)$.

Furthermore, we calculated the proportion of cases with mutated sex chromosomes in different tumor entities, i.e. those who did not have the normal XX or $\mathrm{XY}$ karyotype. The comparison between the proportion of mutant sex chromosomes in tumor entities of women and men was then represented as a curve diagram (Fig. 3). 


\subsection{Survival analysis and statistics}

The data from the ploidy analysis and the calculated proportion of mutated sex karyotypes were then correlated with the 5-year survival rates by calculating regression/ correlation coefficients using regression analysis provided by Excel (Microsoft Office Excel 2007).

\section{Results}

\subsection{Gender-specific survival}

Table 1 shows the results of the literature research for gender-specific survival. We examined 27 tumors that occur in both sexes, and seven gender-specific malignancies. According to the number of studies, we characterized the gender-specific prognosis of the individual tumors. Furthermore, we recorded the 5-year survival rates.

It is apparent that for more than $50 \%$ of the tumor entities, the studies showed a significantly better survival for women. For instance in malignant melanoma, gastric cancer and esophageal cancer, all studies reported a survival advantage of women. Also in lung cancer, all studies except one indicated a better prognosis of women.

But there were also entities in which the majority of studies have noted no difference in survival, e.g. in urinary bladder carcinoma and osteosarcoma.

In a third group of tumors the available data was not conclusive, the number of trials with similar survival times in men and women were equal to those reporting a survival advantage of women.

Overall, just 4 out of 68 analyzed studies reported a survival advantage of men. Specifically, this was observed for gall bladder cancer, acute lymphocytic leukemia, urinary bladder and oral cavity cancer (Table 1).

\subsection{Tumor ploidy}

In this part of the study the total modal chromosome numbers of tumors were analyzed and their frequency distributions represented by a histogram chart (Fig. 1). The data was retrieved from the Mitelman database of chromosomal alterations in cancer. In total, 36.859 karyograms were analyzed. Based on the histograms it became evident which chromosome numbers and thus which ploidy level were most frequently present in the different tumor entities. Representations of colon cancer, chronic myeloid leukemia, osteosarcoma and testicular tumors are shown in Fig. 1. The histograms of all other evaluated tumor entities are available as supplementary data (supplementary figure 1).

All tumor entities, except testicular tumors, showed a clear peak of chromosome numbers in the peridiploid range. In several entities, this was actually the only peak in the distribution. In AML, CLL, salivary gland tumors and thyroid carcinomas the tumors were peridiploid in the overwhelming number of cases. Also CML, ALL, B-cell lymphomas, the fibroblastic/myofibroblastic tumors, liposarcoma, Wilms tumor, as well as multiple myeloma showed a singular diploid peak, but with a somewhat more diffuse distribution and the presence of hyperdiploid and hypodiploid cases. The penis and prostate tumors showed also these peridiploid peaks, but moreover an accumulation of chromosome numbers in the tetraploid range was observed.

Several tumors showed chromosome numbers in the triploid range, as exemplified in the Fig. 1 for colon carcinoma and osteosarcoma. Other tumor types of this group were esophageal carcinoma, gastric carcinoma, gallbladder carcinoma and lung carcinoma. Also pancreatic carcinoma, leiomyosarcoma, as well as ovarian cancer showed a similar distribution which was also detectable in Hodgkin lymphoma, malignant fibrous histiocytoma and cervical cancer.

In addition, there is a group of tumors which had chromosome numbers in the triploid range but without a clear peak in the frequency distribution. This group includes renal tumors, malignant melanoma, bladder carcinoma, hepatocellular carcinoma, GIST, astrocytoma grade III-IV, endometrial tumors, breast cancer and the fibrohistiocytic tumors.

Very unusual is the distribution of chromosome numbers of testicular tumors which are actually never peridiploid but instead showed a peak between the diploid and triploid range (Fig. 1).

We did not analyze the karyograms of 37 tumor types in the Mitelman database for specific karyotypic changes, but it was noted that even within the group of diploid tumors (No. of chromosome $=46$ ) there was not a single karyotype without at least one structural or nummerical aberration.

\subsection{Correlation between ploidy and survival}

Using a regression analysis, the extent of chromosomal changes in various tumor entities were compared with the published survival rates. Specifically, the number of cases with hyperdiploid, predominant triploid, predominant hypertriploid and aneuploid chromosome sets were calculated and correlated with the 5-year survival rates. The calculated regression/correlation coefficients showed a significant association between a survival decrease and an increase of aberrant chromosome numbers in carcinomas (Fig. 2). In the analysis of all tumor entities, no statistically significant correlation could be demonstrated.

\subsection{Analysis of changes in the sex chromosomes}

In Fig. 3, the percentages of mutated sex chromosomes were represented for those tumor entities that occur in both 


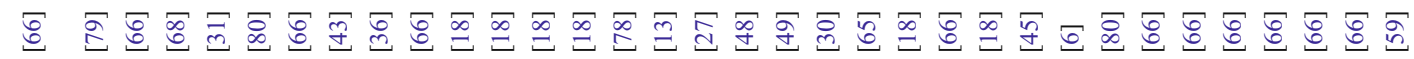

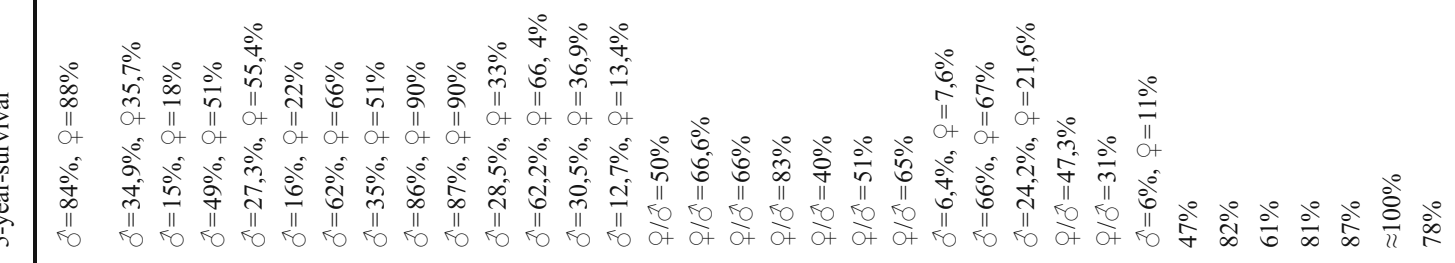

三

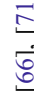

守

主

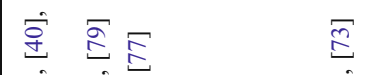

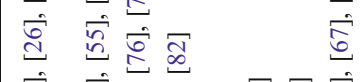

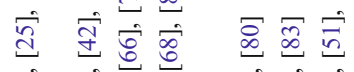

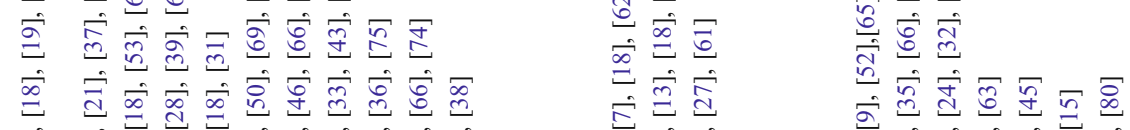

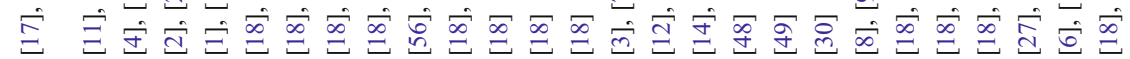
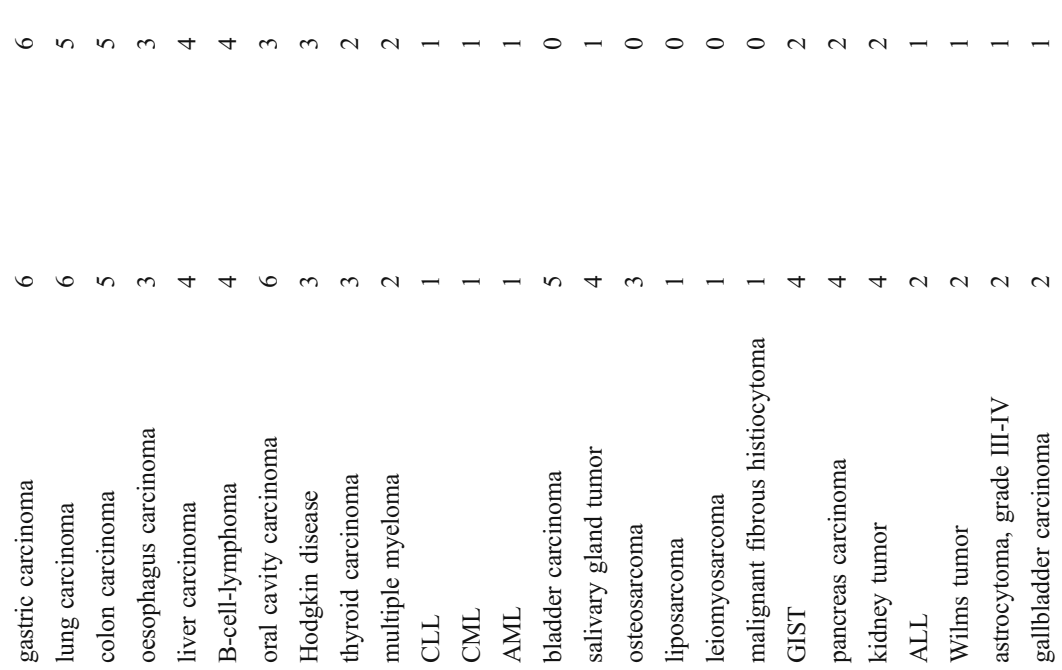

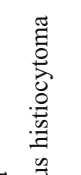

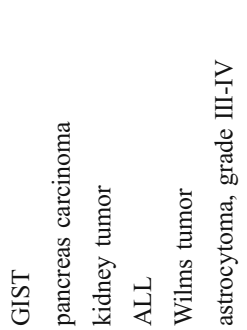

\section{至}

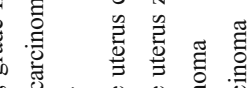

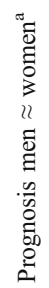

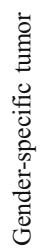


osteosarcoma $(n=176)$

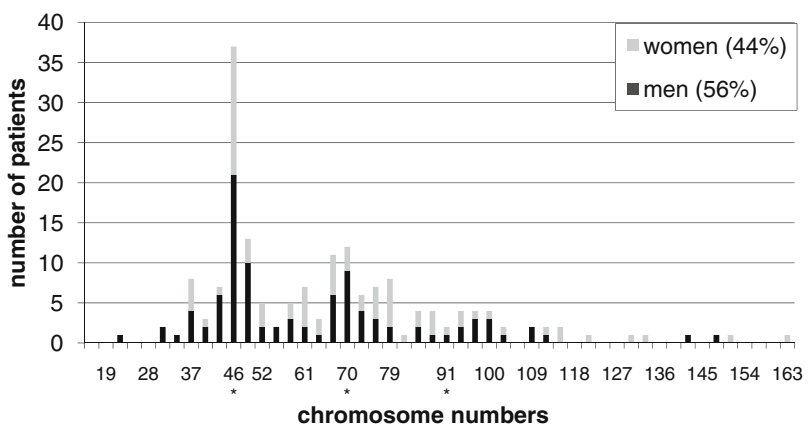

testis tumor $(n=343)$

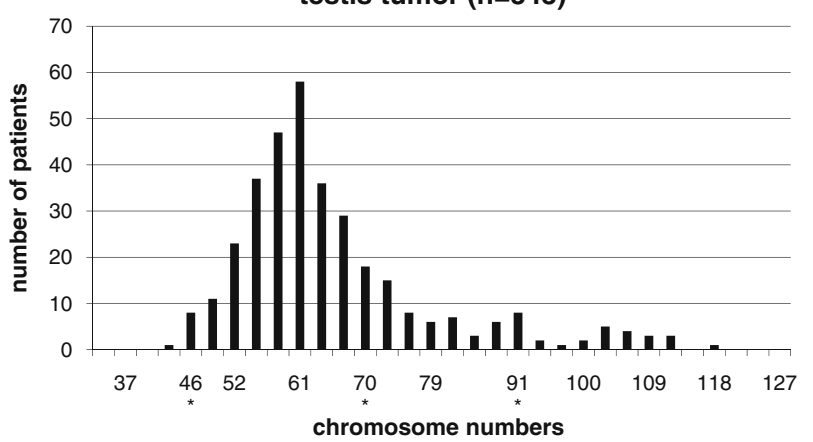

CML ( $\mathrm{n}=3397)$
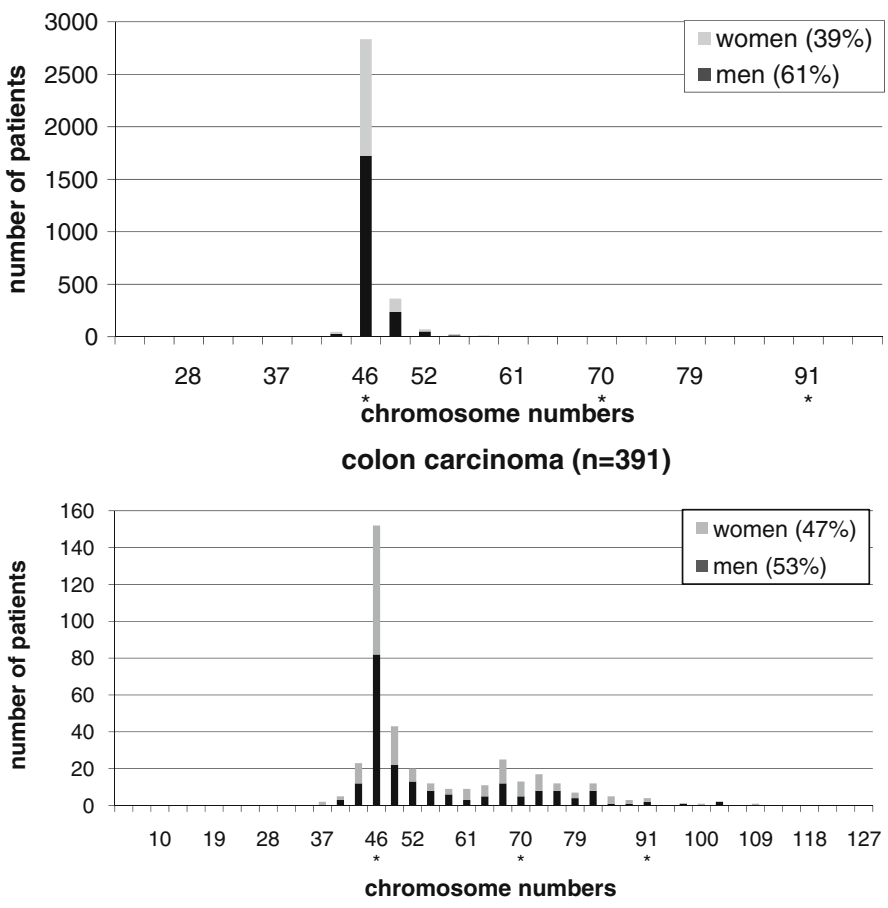

Fig. 1 Examples for ploidy analysis of tumor entities. Histograms showing the frequency of chromosome numbers in tumor entities for men and women

women and men. It became evident that the percentage of numerical sex chromosome aberrations is generally higher in men compared to women suggesting a potential association with the gender specific difference in survival. However, when correlating the percentage differences of the mutated sex karyotypes with differences in 5-year survival rates between women and men, no statistically significant relationship could be established.

\section{Discussion}

\subsection{Differences in gender-specific survival}

One aim of this study was to create an overview of the gender-specific survival in malignant tumors. One earlier study already presented data for malignant melanoma, soft tissue sarcoma and cancers of the breast, lung, colorectal, esophagus, gastric, pancreatic and soft tissue [47]. Except for pancreas and breast cancer a better survival of women could be demonstrated. Apart from many individual reports which examined gender as a possible prognosis parameter among many others, there are only few comprehensive studies which deal with this issue in multiple tumor entities. Noteworthy in this context is the Eurocare-3-study and for Germany the data of the Robert Koch Institute (RKI).
The Eurocare-3 study examined among other things the gender-specific survival of 42 different malignancies in adults and from 24 malignant tumors in children. Overall, the study included 1.8 million adults and 24,000 children from 20 European countries who were diagnosed with cancer during the period from 1990 to 1994 . The patients were observed 5 years after diagnosis until 1999. Various factors were compared with each other, for example the survival between countries, the survival among the different tumors and the survival of women and men. It was recognized that for most tumors, women had a significantly better survival [18]. The results of the Robert Koch Institute represent retrospectively collected data being based on the information from the tumor registries of individual states of Germany. Again, an overall survival advantage of women for most tumor entities could be demonstrated [66]. To our knowledge, this is the first study which primarily focused on this issue and analyzed a large number of tumor types with compilation of data from various reports to provide an estimate of the evidence for gender specific difference in cancer survival.

In the literature the following possible causes for the differences in gender-specific survival are mentioned: differences in tumor biology, defense mechanisms, perception of symptoms and stage at the time of diagnosis. Generally it is stressed that women live healthier, attend 

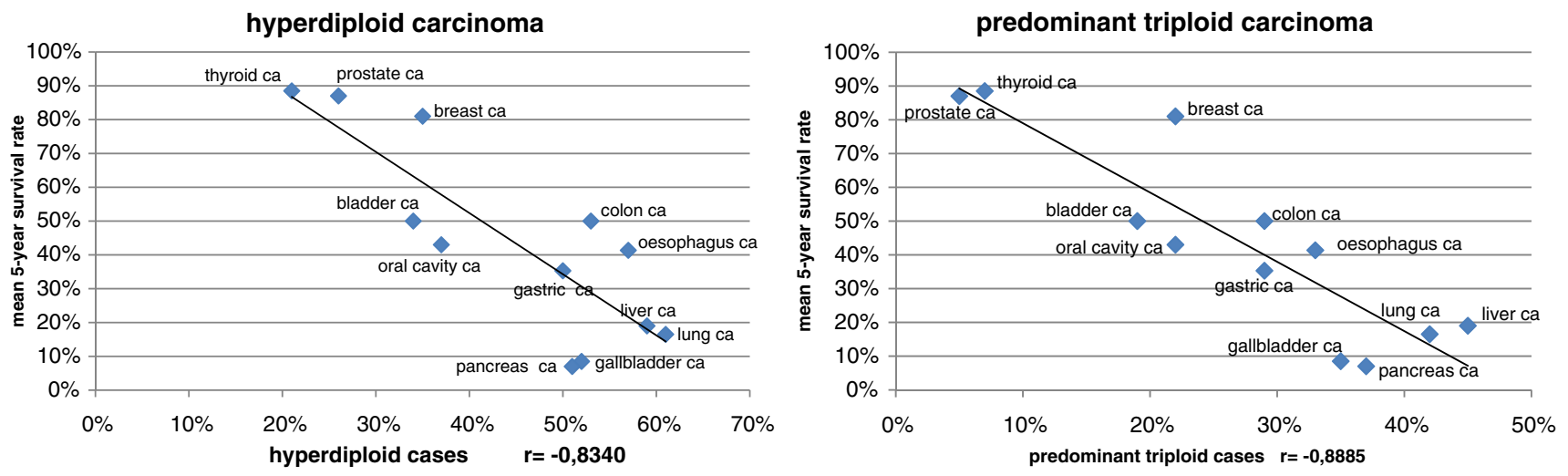

predominant hypertriploid

carcinoma
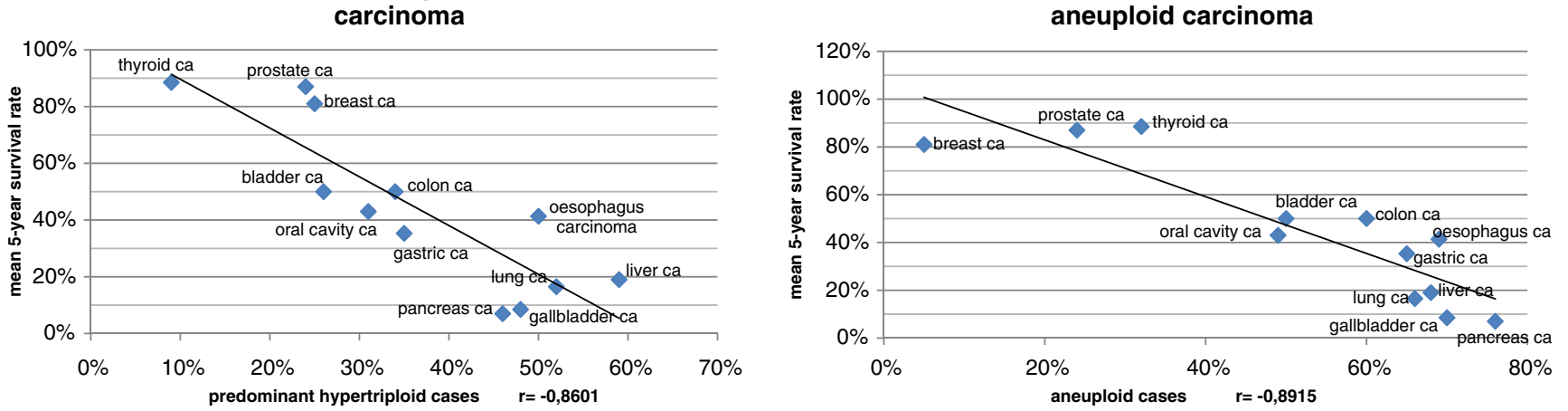

Fig. 2 Correlation between the extent of (aneu)ploidy states and mean 5 -year survival rate in different carcinomas. Each point correspond to one carcinoma entity as indicated. The survival rates were correlated with the percentage of cases within each entity showing hyperdiploid

(No.chr. $>47)$, predominant hypertriploid (No.chr $>57$ ), predominant triploid (No.chr. 57-83) and aneuploid (No.chr. $\neq 45-47, \neq 90-92$ ) chromosome numbers. The correlation coefficients (r) indicate significant correlations between these (aneu)ploidy states and the 5-year survival rates

more often screening programs and look for medical advice earlier than men. Thereby tumors may get detected and treated at an earlier stage of the disease.

However, many studies taking into account tumor stage also demonstrated that gender remains a significant and independent prognostic factor. This could be shown, for example, in malignant melanoma [64], colorectal [82] and lung cancer [70]. Thus, it must be postulated that tumorbiological parameters being associated with the sex of the patient are responsible for the survival advantage of women. In addition, the better overall life expectancy of women need to be considered in this context.

\subsection{Ploidy and survival}

Another objective of this study was to investigate the ploidy level of tumors. This was done by analyzing data from the Mitelman database allowing the assessment of the number of chromosomes in various tumor entities [44]. A similar approach was performed recently by Storchova and Kuffer [72]. These authors, however, placed their emphasis on the analysis of tetraploidy. In our study, particular attention was given to the analysis of triploidy, i.e. tumors exhibiting chromosome numbers in the triploid range, and the question

whether this state has any tumor-biological relevance with respect to cancer survival and gender-specific prognosis.

Our analysis indicated that many tumor entities have chromosome numbers with a bimodal distribution with a peak in the triploid range in addition to a diploid one. This was observed particularly in tumors with a high mortality like lung, pancreas, gastric and colon cancer. For lung cancer, we recently showed that a near triploid DNA content is frequently detectable in non-small cell carcinomas [60]. In fact, Ozaki et al. already reported in 1981 that near-triploidy represent the most frequent DNA distribution in lung cancer [54]. Thus, triploidy is not a cell culture artifact. The observation of tripolar mitoses was indicative for a DNA peak in the triploid range [60]. Based on the correlation analysis with survival we could show that the detection and in particular the extent of triploidy is associated with poor survival in carcinomas. Triploidy in this context is obviously correlated with aneuploidy.

In an earlier study by DNA cytometry the bimodal distribution of chromosome numbers was shown for many tumors [5]. The authors already pointed to the influence of the modal DNA value and the ploidy level on prognosis. For the vast majority of tumors, with the exception of squamous cell carcinoma of the cervix uteri, a survival 


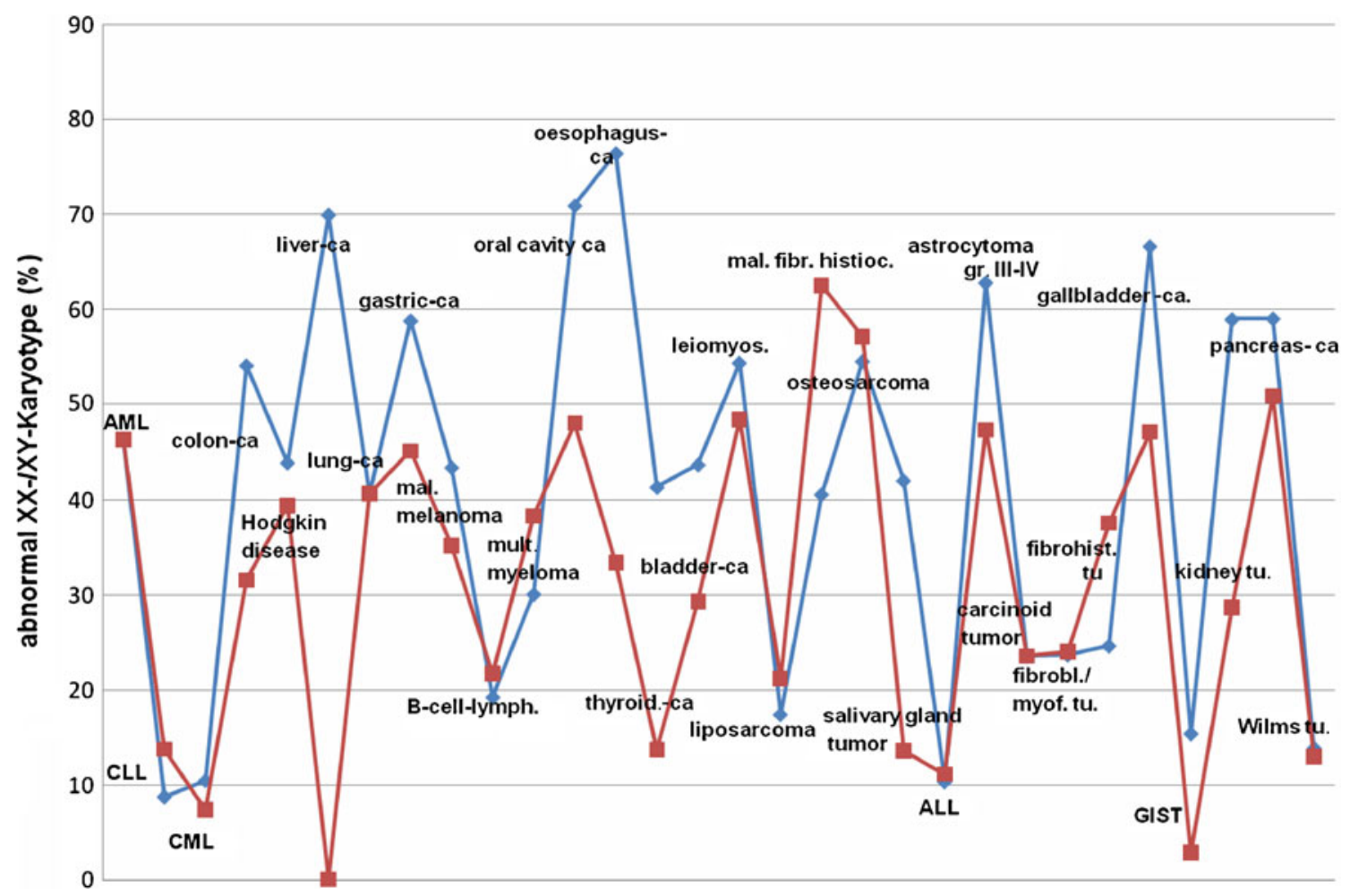

Fig. 3 Analysis of percentage of cases with XX or XY numerical alterations in the different tumor entities (blue colour: percentage of cases in men with XY numerical alterations; red colour: percentage of cases in women with XX numerical alterations)

benefit was observed for the near diploid tumors compared to those in the triploid-tetraploid range [5]. Our study confirmed that the extent of abnormal chromosome numbers in carcinomas is associated with worse prognosis (Fig. 2). This finding again highlights the importance of aneuploidy and chromosomal chaos for cancer survival. In addition it suggests that quantifying the amount of nondiploid cells in carcinomas might be used as a prognostic marker.

The data for non-carcinoma tumor types is heterogeneous. At least there is not such a clear association between the extent of triploidy or aneuploidy with survival. In this context, the analysis of Kaneko and Knudson in neuroblastoma is interesting. It is one of the few reports that dealt with the relevance of ploidy level on tumor behavior. The authors demonstrated that neuroblastoma in lower tumor stages and with improved survival more frequently had a near triploid chromosome set while the near diploid tumors were characterized by advanced stages and poor survival [34].

Tripolar mitosis and triploidy are also detectable in normal cells and are associated with each other as was already shown more than 40 years ago. Tripolar mitoses represent a subtype of multipolar mitoses and occur usually in octaploid or tetraploid cells. During cell division these cells generate triploid and haploid caughter cells $(8 n$ : $3 n+$ $3 n+2 n, 4 n: 3 n+1 n)$. However it is a rare phenomenon that occurred in only $3,6 \%$ of the analyzed mitoses [57], [58]. In malignancies, in contrast, cells with a near triploid chromosome content frequently represent the predominant tumor cell clone. And this may be reflected microscopically by the presence of tripolar mitoses. Thus, triploidy probably represents a metastable condition of the chromosome complement.

Our study revealed profound differences in the incidence of triploidy. In some entities it is detectable very often while being almost absent in others. This may have practical importance in the differential diagnosis of malignant undifferentiated tumors of unknown primary site. The detection of a triploid tumor cell clone by DNA measurement or the observation of tripolar mitoses may provide important information in this regard.

Testicular tumors revealed a peculiar histogram of the chromosome number because it did not show the typical peak in the diploid range. This was also observed by DNA cytometry [5]. It can only be speculated whether this distribution has any biological or clinical relevance [23]. In this context we would like to mention that germ cell tumors belong to those entities which frequently show good response to chemotherapy and even spontaneous tumor regression [10]. The histogram suggests that the chromosomes numbers of germ cell tumor may be more frequently in an unstable state between diploidy and triploidy which may be associated with the above mentioned phenomena. 
By analyzing the 36.859 karyograms of the Mitelman database we did not find a single case with normal chromosome number $(n=46)$ without at least one structural or nummerical aberration suggesting that chromosomal changes are an inherent feature of cancer formation.

Another observation of interest was the fact that numerical aberrations of the sex chromosomes are more prevalent in tumors from male compared to female patients. The imbalance in relation to the two different gonosomes in men may lead to an increased chromosomal instability. This, in turn, may predispose to the accumulation of additional chromosomal changes as the major cause for tumor progression and cancer related death. Our observation and the above mentioned hypothesis represents a possible biological cause for the phenomenon of worse survival in men. However, it should be noted that the difference in the number of changes of the sex chromosomes in the different tumor entities could not be significantly associated with the difference in the genderspecific survival rates. Apart from alterations of the sex chromosomes, other factors probably play a role within individual tumor types.

In conclusion, a significant survival advantage of women could be shown for most cancer types. Triploidy is a common phenomenon in many but not all tumor entities, its extent is associated with poor prognosis in carcinomas. Tumors of male patients more frequently harbour numerical alterations of the sex chromosomes than tumors of females.

We suggest that the biological mechanisms underlying gender specific differences in survival should be further investigated. Also the role of triploidy in cancer biology and progression merits more attention. Finally, our study again highlights the fundamental importance of chromosomal changes in tumor biology.

Acknowledgement The support of Dr. Annelore Altendorf-Hofmann and the Jena cancer registry is gratefully acknowledged.

Open Access This article is distributed under the terms of the Creative Commons Attribution Noncommercial License which permits any noncommercial use, distribution, and reproduction in any medium, provided the original author(s) and source are credited.

\section{References}

1. L.A. Anderson et al., Mortality in Barrett's oesophagus: results from a population based study. Gut 52(8), 1081-1084 (2003)

2. E. Angell-Andersen et al., Colorectal cancer survival trends in Norway 1958-1997. Eur. J. Cancer 40(5), 734-742 (2004)

3. A.A. Antunes et al., Analysis of prognostic factors in patients with transitional cell carcinoma of the bladder treated with radical cystectomy. Int. Braz. J. Urol. 32(1), 35-42 (2006)

4. H. Asamura et al., A Japanese lung cancer registry study: prognosis of 13, 010 resected lung cancers. J. Thorac. Oncol. 3 (1), 46-52 (2008)
5. N.B. Atkin, R. Kay, Prognostic significance of modal DNA value and other factors in malignant tumours, based on 1465 cases. Br. J. Cancer 40(2), 210-221 (1979)

6. AWMF online, Arbeitsgemeinschaft der Wissenschaftlichen Medizinischen Fachgesellschaften, Leitlinien für Diagnostik und Therapie in der Neurologie; 3. überarbeitete Auflage 2005, ISBN3-13-132413-9; Georg Thieme Verlag Stuttgart

7. R.A. Badalament et al., Prognostic factors in patients with primary transitional cell carcinoma of the upper urinary tract. J. Urol. 144 (4), 859-863 (1990)

8. Y.K. Bai et al., Analysis of prognostic factors in gastrointestinal stromal tumors of the small intestine. Zhonghua Wei Chang Wai Ke Za Zhi 8(3), 213-216 (2005)

9. Y.K. Bai et al., Multivariate analysis of prognosis in gastrointestinal stromal tumor. Zhonghua Zhong Liu Za Zhi 27(10), 598-601 (2005)

10. B.L. Balzer, T.M. Ulbright, Spontaneous regression of testicular germ cell tumors: an analysis of 42 cases. Am. J. Surg. Pathol. 30 (7), 858-865 (2006)

11. A. Barchielli et al., Long-term prognosis of gastric cancer in a European country: a population-based study in Florence (Italy). 10-year survival of cases diagnosed in 1985-1987. Eur. J. Cancer 37(13), 1674-1680 (2001)

12. N. Bhattacharyya, Survival and prognosis for cancer of the submandibular gland. J. Oral Maxillofac. Surg. 62(4), 427-430 (2004)

13. N. Bhattacharyya, M.P. Fried, Determinants of survival in parotid gland carcinoma: a population-based study. Am. J. Otolaryngol. 26(1), 39-44 (2005)

14. S.S. Bielack et al., Prognostic factors in high-grade osteosarcoma of the extremities or trunk: an analysis of 1,702 patients treated on neoadjuvant cooperative osteosarcoma study group protocols. J. Clin. Oncol. 20(3), 776-790 (2002)

15. S.H. Bigner et al., Specific chromosomal abnormalities in malignant human gliomas. Cancer Res. 88, 405-411 (1988)

16. A. Böcking, Biologische Grundlagen und Anforderungen an die Methodik der statischen DNA-Zytometrie, Fortschritt und Fortbildung in der Medizin 21 (1997/98), Deutscher Ärzte-Verlag, Bundesärztekammer (Hrsg.), Köln, pp. 21-40, ISSN 0170-3331

17. P.G. Buettner et al., Development of Prognostic Factors and Survival in Cutaneous Melanoma over 25 Years: An Analysis of the Central Malignant Melanoma Registry of the German Dermatological Society, Wiley InterScience (www.interscience. wiley.com), (2005)

18. M.P. Coleman et al., EUROCARE-3 summary: cancer survival in Europe at the end of the 20th century. Ann. Oncol. 14 (Supplement 5), v128-v149 (2003)

19. E. Crocetti et al., Prognostic variables and prognostic groups for malignant melanoma. The information from Cox and classification and regression trees analysis: an Italian population-based study. Melanoma Res. 16(5), 429-433 (2006)

20. P. Duesberg, R. Li, A. Fabarius, R. Hehlmann, The chromosomal basis of cancer. Cell. Oncol. 27(5-6), 293-318 (2005)

21. V. Fontana et al., Socioeconomic status and survival of gastric cancer patients. Eur. J. Cancer 34(4), 537-542 (1998)

22. M.L. Friedlander et al., Clinical and biological significance of aneuploidy in human tumours. J. Clin. Pathol. 37(9), 961-974 (1984)

23. A. Frigyesi et al., A model for karyotypic evolution in testicular germ cell tumors, genes. Chromosom. Cancer 40, 172-178 (2004)

24. Y. Fuji et al., Prognostic factors of renal cell carcinoma: a multivariate analysis. Hinyokika Kiyo 42(2), 85-89 (1996)

25. C. Garbe, A. Blum, Epidemiology of cutaneous melanoma in Germany and worldwide. Skin Pharmacol. Appl. Skin Physiol. 14, 280-290 (2001)

26. C. Garbe, C.E. Orfanos, Epidemiology of malignant melanoma in central Europe: risk factors and prognostic predictors. Results of the 
Central Malignant Melanoma Registry of the German Dermatological Society, Pigment Cell Res. (Suppl 2), 285-94 (1992)

27. G. Gatta et al., EUROCARE-3: childhood cancer survival in Europe. Ann. Oncol. 14 (Supplement 5), v119-v127 (2003)

28. S. Greve, Rektumkarzinom. Chirurgische Therapie-ChemotherapieStrahlentherapie; Langzeitergebnisse", Inaugural-Dissertation, Johann Wolfgang Goethe-Universität Frankfurt am Main, (2003)

29. G. Haroske, J.P. Baak, H. Danielsen, F. Giroud, A. Gschwendtner, M. Oberholzer, A. Reith, P. Spieler, A. Böcking, Fourth updated ESACP consensus report on diagnostic DNA image cytometry. Anal. Cell. Pathol. 23, 89-95 (2001)

30. J. Heinz, Sarkome, Vorlesung, (2008)

31. H. Hidaka et al., Sex difference in survival of patients treated by surgical resection for esophageal cancer. World J. Surg. 31(10), 1982-1987 (2007)

32. H. Inomiya et al., Prognostic factors in patients with nonmetastatic renal cell carcinoma. Nippon Hinyokika Gakkai Zasshi 87(9), 1099-1104 (1996)

33. D. Kademani et al., Prognostic factors in intraoral squamous cell carcinoma: the influence of histologic grade. J. Oral Maxillofac. Surg. 63(11), 1599-1605 (2005)

34. Y. Kaneko, A.G. Knudson, Mechanism and relevance of ploidy in neuroblastoma. Genes Chromosom. Cancer 29(2), 89-95 (2000)

35. Kaspar et al., Das Pankreaskarzinom: Aktuelle Epidemiologie, Diagnostik, Therapie, Onkologie 4/2006:14-22

36. B. Klimm et al., Role of hematotoxicity and sex in patients with Hodgkin's lymphoma: an analysis from the German Hodgkin study group. J. Clin. Oncol. 23(31), 8003-8011 (2005)

37. D.A. Kooby et al., Biologic predictors of survival in nodenegative gastric cancer, Ann. Surg. Vol. 237, No. 6, 828-837

38. S.Y. Kristinsson et al., Patterns of survival in multiple myeloma: a population-based study of patients diagnosed in Sweden from 1973 to 2003. J. Clin. Oncol. 25(15), 1993-1999 (2007)

39. M. Kux et al., Results of surgery for colon carcinoma. Eur. Surg. 19(4), 583-587 (1987)

40. K.G. Lasithiotakis et al., The Incidence and Mortality of Cutaneous Melanoma in Southern Germany: Trends by Anatomic Site and Pathologic Characteristics 1976 to 2003, Wiley InterScience (www.interscience.wiley.com), (2006)

41. F. Levi et al., Prognostic factors for cutaneous malignant melanoma in Vaud, Switzerland. Int. J. Cancer 78(3), 315-319 (1998)

42. A. Maguire et al., Sex as a prognostic factor in gastric cancer. Eur. J. Cancer 32A(8), 1303-1309 (1996)

43. S. Mak-Kregar et al., A nationwide study of the epidemiology, treatment and survival of oropharyngeal carcinoma in The Netherlands. Eur. Arch. Otorhinolaryngol. 252(3), 133-138 (1995)

44. Mitelman Database of Chromosome Aberrations and Gene Fusions in Cancer (2010). Mitelman F, Johansson B and Mertens F (Eds.), http://cgap.nci.nih.gov/Chromosomes/Mitelman

45. E. Mitry et al., Incidence of and survival from Wilms' tumour in adults in Europe: data from the EUROCARE study. Eur. J. Cancer 42(14), 2363-2368 (2006)

46. T. Mitterlechner et al., Epidemiology of non-Hodgkin lymphomas in Tyrol/Austria from 1991 to 2000. J. Clin. Pathol. 59(1), 48-55 (2006)

47. R. Molife, P. Lorigan, S. MacNeil, Gender and survival in malignant tumours. Cancer Treat. Rev. 27(4), 201-209 (2001)

48. K. Moore Dalal et al., Subtype specific prognostic nomogram for patients with primary liposarcoma of the retroperitoneum, extremity, or trunk. Ann. Surg. 244(3), 381-391 (2006)

49. E. Morera Serna, Laryngeal leiomyosarcoma. Acta Otorrinolaringol. Esp. 58(9), 445-448 (2007)

50. N. Nagasue et al., Better survival in women than in men after radical resection of hepatocellular carcinoma. Hepatogastroenterology 36 (5), 379-383 (1989)
51. A. Nieto, M.R. Ramos, Rising trends in oral cancer mortality in Spain, 1975-94. J. Oral Pathol. Med. 31(3), 147-152 (2002)

52. Y.W. Novitsky et al., Long-term outcomes of laparoscopic resection of gastric gastrointestinal stromal tumors. Ann. Surg. 24(6), 738-745 (2006). discussion 745-7

53. S.H. Ou et al., Prognostic factors for survival of stage I nonsmall cell lung cancer patients: a population-based analysis of 19, 702 stage I patients in the California Cancer Registry from 1989 to 2003. Cancer 110(7), 1532-1541 (2007)

54. H.S. Ozaki et al., Nuclear DNA modal patterns as diagnostic criteria in human lung cancer. Jpn J. Clin. Oncol. 11(3), 431-442 (1981)

55. D. Palli et al., Dietary and familial determinants of 10-year survival among patients with gastric carcinoma. Cancer 89(6), 1205-1213 (2000)

56. D. Papathanassiou, Prognosefaktoren des papillären Schilddrüsenkarzinoms Ergebnisse der Chirurgischen Universitätsklinik Düsseldorf im Vergleich mit international eingesetzten Scoresystemen, Dissertation, Heinrich-Heine-Universität Düsseldorf, (2006)

57. F. Pera, B. Rainer, Studies of multipolar mitoses in euploid tissue cultures. I. Somatic reduction to exactly haploid and triploid chromosome sets. Chromosoma 42(1), 71-86 (1973)

58. F. Pera, H.G. Schwarzacher, Distribution of chromosomes to the daughter cell nuclei of multipolar mitoses in euploid tissue cultures of Microtus agrestis. Chromosoma 26(3), 337-354 (1969)

59. B. Perez et al., Squamous carcinoma of the penis. Arch. Esp. Urol. 53(8), 693-699 (2000)

60. I. Petersen et al., Core classification of lung cancer: correlating nuclear size and mitoses with ploidy and clinicopathological parameters. Lung Cancer 65(3), 312-318 (2009)

61. P. Picci, Osteosarcoma (osteogenic sarcoma). Orphanet J. Rare Dis. 23, 2-6 (2007)

62. K.S. Png et al., Prognostic factors for upper tract transitional cell carcinoma: a retrospective review of 66 patients. Asian J. Surg. 31 (1), 20-24 (2008)

63. C.H. Pui et al., Childhood and adolescent lymphoid and myeloid leukemia, Hematology Am Soc Hematol Educ Program. 118-45 (2004)

64. D.S. Reintgen et al., Sex related survival differences in instances of melanoma. Surg. Gynecol. Obstet. 159(4), 367-372 (1984)

65. K.K. Richter et al., Long-term follow-up in 54 surgically treated patients with gastrointestinal stromal tumours. Langenbecks Arch. Surg. 393(6), 949-955 (2008)

66. Robert-Koch-Institut (Hrsg) und die Gesellschaft der epidemiologischen Krebsregister in Deutschland e.V. (Hrsg)., Krebs in Deutschland 2003-2004. Häufigkeiten und Trends., 6. überabeitete Auflage. Berlin, (2008)

67. E.M. Ruggeri et al., Long-term survival in locally advanced oral cavity cancer: an analysis of patients treated with neoadjuvant cisplatin-based chemotherapy followed by surgery. 27(6), 452-8 (2005)

68. M. Sant et al., EUROCARE-3: survival of cancer patients diagnosed 1990-94-results and commentary. Ann. Oncol. 14 (Supplement 5), v61-v118 (2003)

69. K. Shibuya, E. Yano, Regression analysis of trends in mortality from hepatocellular carcinoma in Japan, 1972-2001. Int. J. Epidemiol. 34, 397-402 (2005)

70. F. Siddiqui et al., The influence of gender, race, and marital status on survival in lung cancer patients analysis of radiation therapy oncology group trials. J. Thorac. Oncol. 5(5), 631-639 (2010)

71. A. Stang et al., Skin melanoma in Saarland: incidence, survival and mortality 1970-1996. Eur. J. Cancer Prev. 10(5), 407-415 (2001)

72. Z. Storchova, C. Kuffer, The consequences of tetraploidy and aneuploidy. J. Cell Sci. 121(Pt 23), 3859-3866 (2008)

73. K. Sundaram et al., Carcinoma of the oropharynx: factors affecting outcome. Laryngoscope 115(9), 1536-1542 (2005)

74. Südwestsächsisches Tumorzentrum Zwickau e.V (Hrsg.), Bösartige Neubildungen der Schilddrüse im Raum Südwestsachsen ICD10: C73, (2008) 
75. J. Swerdlow, Epidemiology of Hodgkin's disease and nonHodgkin's lymphoma. Eur. J. Nucl. Med. Mol. Imaging 30 (Suppl 1), S3-S12 (2003)

76. L. Teppo et al., Cancer patient survival-patterns, comparisons, trends-a population-based Cancer Registry study in Finland. Acta Oncol. 38(3), 283-294 (1999)

77. J. Tsurutami et al., Prognostic significance of clinical factors and Akt activation in patients with bronchioloalveolar carcinoma. Lung Cancer 55(1), 115-121 (2007)

78. G Wegener, Klinisch-epidemiologische Daten zum Harnblasenkarzinom, Vorlesung, Medizinische Hochschule Hannover, Tumorzentrum Hannover, (2003)
79. Tumorregister München (Hrsg), Überleben C16: Magenkarzinom, www.tumorregister-muenchen.de, surv_C16__G.pdf, (2007)

80. Tumorzentrum Jena (Koordination), Überleben nach KrebsSachbericht der Tumorzentren in Thüringen, Maligne solide Organtumoren 1996-2005, (2007)

81. F.A. Wenger et al., Prognosis factors in carcinoma of the head of the pancreas. Dig. Surg. 17(1), 29-35 (2000)

82. U. Wolters et al., Colorectal cancer-a multivariate analysis of prognostic factors. Eur. J. Surg. Oncol. 22(6), 592-597 (1996)

83. www.nhl-info.de/exec/start?site=/non_hodgkin_lymphome/465. htm\&check=0, Dr. Michael Kübrich (Projektleitung), NonHodgkin-Lymphome, Verlauf, ribosepharm division (Impressum) 